\title{
Transmission Electron Microscopy Study of Low Mo-content Bi-Mo-O Phases
}

\author{
Á. R. Landa-Cánovas*, Eladio Vila*, Jorge Hernández-Velasco*, Jean Galy** and Alicia Castro* \\ * Instituto de Ciencia de Materiales de Madrid, ICMM, CSIC, Sor Juana Inés de la Cruz 3, \\ Cantoblanco, 28049 Madrid, Spain \\ ** Centre d'Elaboration de Matériaux et d'Etudes Structurales CNRS, 29 rue Jeanne Marvig, BP \\ 94347, 31055 Toulouse CEDEX 4, France
}

$\delta$-Bi2O3, a material with a fluorite-type structure, is one of the best solid-state oxygen-ion conductors. It is a high-temperature form that cannot be quenched to room temperature. However, doping with small amounts of transition metal oxides preserves the $\delta$ - $\mathrm{Bi} 2 \mathrm{O} 3$ structure at low temperature and retains its anionic conduction properties. The $\mathrm{Bi} 2 \mathrm{O} 3-\mathrm{MoO} 3$ materials are interesting because of their functional properties, chiefly as catalysts and as good ionic conductors. All the phases in this system are related to the fluorite structure except Bi2MoO6 which shows an Aurivillius-type structure.

Buttrey et al. [1] first reported the compound Bi38Mo7O78 as a $5 \times 3 \times 3$ fluorite-type $\delta$-Bi2O 3 superstructure based on electron diffraction experiments. Sharma et al. [2] resolved the structure by single crystal X-ray diffraction with space group $\mathrm{Pbcn}$. The structure consists of fluorite-type OBi4 groups with the remaining oxygens forming $\{\mathrm{MoO} 4\}$ tetrahedra and $\{\mathrm{MoO} 6\}$ octahedra. Later on, Kuang et al. [3] reported the composition range $\mathrm{Bi} 38-\mathrm{xMo} 7+\mathrm{xO} 78+1.5 \mathrm{x}$ for this phase presenting a more-complex $5 \times 3 \times 6$ superstructure with a slight monoclinic distortion. All these phases are high temperature phases and present commensurate $\delta$-Bi2O3 superstructures. In this work we present TEM studies of low-temperature phases prepared by n-butylamine procedure [4-6] the with compositions close to Bi38Mo7O78 showing incommensurate modulations.

In Figure 1 we present a selected area electron diffraction (SAED) pattern from the high-temperature Bi38Mo7O78 commensurate phase showing neat fluorite super-structure reflections. However, SAED patterns (Figures. 2, 3 and 4) from the low-temperature synthesized phases show clearly incommensurate satellites which run slightly away from the [560] fluorite direction. Therefore, the modulation vector is close to $\mathbf{q} \sim 1 / 9\left(\begin{array}{lll}10 & 12 & 0\end{array}\right)^{*}$ and then the diffraction pattern can be indexed as $\mathbf{G}=$ $\mathbf{H} \pm \mathbf{m q}$, where $\mathbf{H}$ is the basic fluorite-type reciprocal lattice, $\mathrm{m}$ an entire number and $\mathbf{q}$ the modulation vector. HRTEM images of the low temperature phases present diffuse waves running "on top" of the basic fluorite-type lattice.

\section{References}

1. Buttrey et al., Mater. Res. Bull. 21:739, 1986

2. Sharma et al., J. Solid-State Chem. 182: 1312, 2009

3. Kuang et al., Chem. Mater. 22: 4484-4494, 2010

4. Vila et al., Journal of Solid State Chemistry 180: 661-669, 2007

5. Landa-Cánovas et al., Acta Crystallographica B65: 458-466, 2009

6. Galy et al., Journal of Solid State Chemistry 182: 1177-1187, 2009 


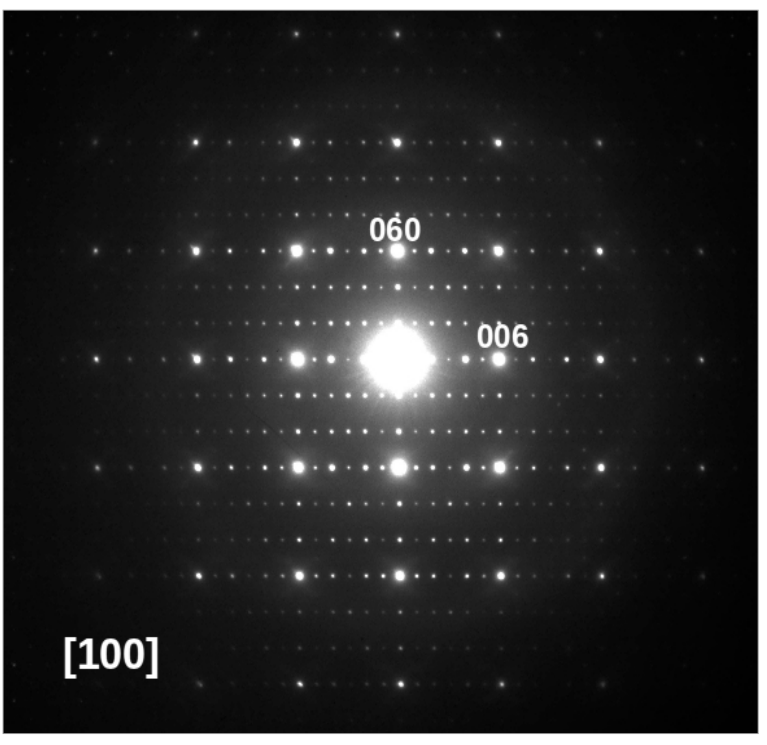

Figure 1. SAED pattern of a commensurate $\mathrm{Bi}_{38} \mathrm{Mo}_{7} \mathrm{O}_{78}$ crystal along [100].

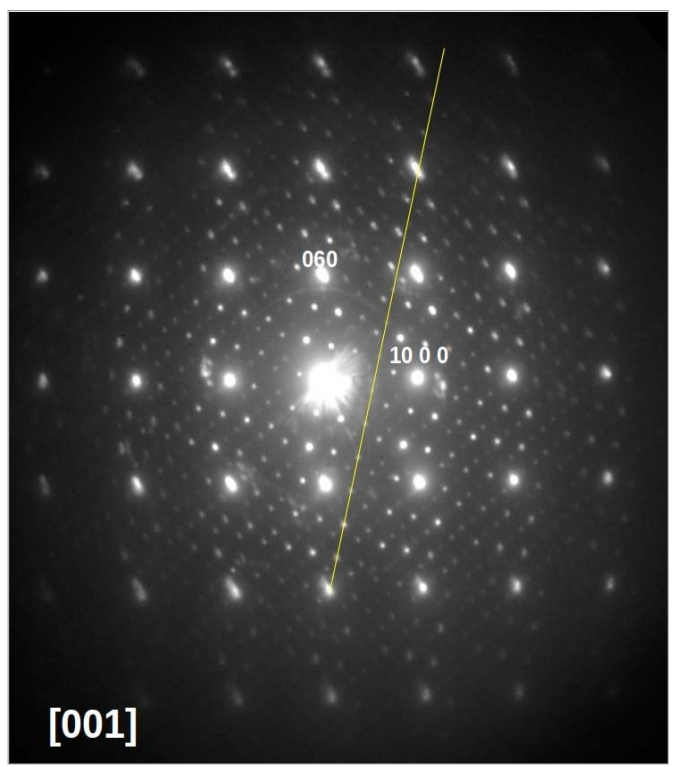

Figure 3. SAED pattern of an incommensurate low-temperature $\mathrm{Bi}_{6} \mathrm{MoO}_{12}$ crystal along [001].

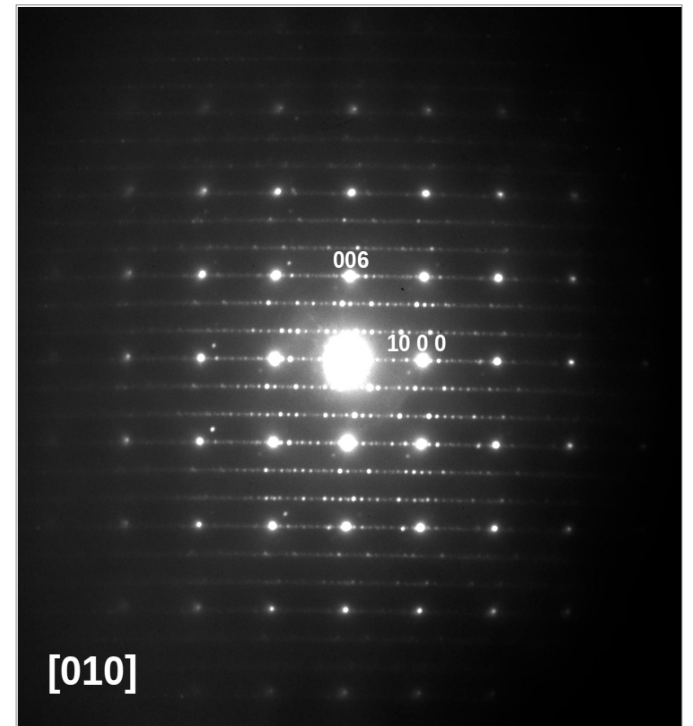

Figure 2. SAED pattern along [010] of an incommensurate low-temperature $\mathrm{Bi}_{14} \mathrm{Mo}_{2} \mathrm{O}_{27}$ crystal.

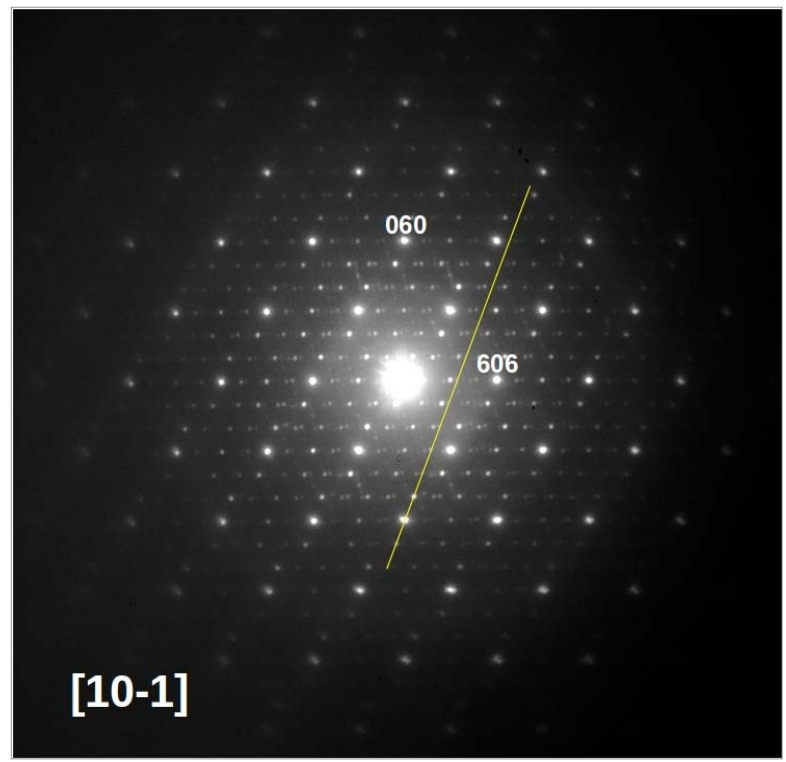

Figure 4. SAED pattern of an incommensurate low-temperature $\mathrm{Bi}_{14} \mathrm{Mo}_{2} \mathrm{O}_{27}$ crystal along [101]. 